\title{
INTERDISCIPLINARIDADE E TRANSDISCIPLINARIDADE NA PESQUISA AGRÍCOLA AMAZÔNICA: UM DESAFIO PARA ATINGIR A SUSTENTABILIDADE
}

\author{
Tatiana Deane de Abreu Sá1; Milton Kanashiro'; Walkymário de Paulo Lemos ${ }^{1}$ \\ ${ }^{1}$ Pesquisadores da Embrapa Amazônia Oriental. Belém, Pará, Brasil. tatiana.sa@embrapa.br
}

\begin{abstract}
Resumo: Este artigo foca em aspectos indicadores de como a pesquisa agropecuária na Amazônia brasileira experimenta um momento de inflexão com tendência de intensificação na demanda de adoção de abordagens interdisciplinares e transdisciplinares, para que efetivamente contribuam ao alcance da sustentabilidade. Partindo de elementos indicadores da diversidade dos tipos de agricultura praticados na região, do histórico e da natureza da pesquisa agropecuária aí praticada, de conceitos inerentes aos tipos de pesquisa quanto ao seu caráter disciplinar, são comentadas estratégias de pesquisa necessárias para abordar um amplo conjunto de grandes temas prioritários e de políticas públicas voltadas ao atingimento da sustentabilidade em empreemdimentos agrícolas, finalizando com a identificação de desafios a enfrentar para ampliar e agilizar a adoção de práticas de pesquisa interdisciplinar e transdisciplinar na Amazônia.
\end{abstract}

PALAVRAS-CHAVE: Amazônia. Ciências agrárias. interdisciplinar.

\section{INTERDISCIPLINARITY AND TRANSDISCIPLINARITY IN AMAZON AGRICULTURAL RESEARCH: A CHALLENGE TO ACHIEVE SUSTAINABILITY}

\begin{abstract}
This article focuses on aspects as indicators of agricultural research in the Brazilian Amazon experiences a turning point with trend intensified demand for adoption of interdisciplinary and transdisciplinary approaches to effectively contribute to achieving sustainability . Leaving indicator elements of diversity of types of agriculture practiced in the region, the history and nature of agricultural research conducted there, the concepts inherent in the types of research regarding its disciplinary character, are discussed research strategies needed to address a broad set of major priority and public policies focused on the achievement of sustainability in agricultural sector, ending with the identification of challenges to expand and accelerate the adoption of practices of interdisciplinary and transdisciplinary research themes in the Amazon.
\end{abstract}

KEYWORDS: Amazon. Agrarian. Interdisciplinary.

\section{TRANSDISCIPLINARIEDAD E INTERDISCIPLINARIEDAD EN LA INVESTIGACIÓN AGRÍCOLA Y AMAZON: UN RETO PARA LOGRAR LA SOSTENIBILIDAD}

RESUMEN: Este artículo se centra en aspectos como indicadores de la investigación agrícola en la Amazonía brasileña experimenta un punto de inflexión con la tendencia se intensificó la 
demanda de adopción de enfoques interdisciplinarios y transdisciplinarios para contribuir eficazmente al logro de la sostenibilidad. Dejando elementos indicadores de la diversidad de los tipos de agricultura que se practican en la región, la historia y la naturaleza de la investigación agrícola realizadas en ellos, los conceptos inherentes a los tipos de investigación con respecto a su carácter disciplinario, se discuten las estrategias de investigación necesarias para abordar un amplio conjunto de las principales prioridades y políticas públicas centrado en el logro de la sostenibilidad en sector emprendimientos agrícola, y que terminan con la identificación de los desafíos a enfrentar para ampliar y acelerar la adopción de prácticas de los temas de investigación interdisciplinarios y transdisciplinarios en la Amazonía.

PALABRAS CLAVE: Amazon. Ciencias agrarias. Interdisciplinarios.

\section{INTRODUÇÃO}

A agricultura, como uma das mais antigas atividades humanas, vem vivenciando uma redefinição revolucionária de sua própria identidade, ao estar sendo impelida a sair de sua habitual ideia monofuncional, de mera supridora de bens para uso e remuneração econômica dos produtores e abastecimento rural e urbano, para abarcar uma ideia multifuncional, em que passa a ser também encarada como supridora de outras funções, tais como, de manejo de recursos naturais renováveis, conservação da paisagem e da biodiversidade, contribuição à viabilidade socioeconômica de áreas rurais e, particularmente, a de prestadora de serviços ecossistêmicos às comunidades, papel esse que vem sendo, inclusive, valorado em escala global (RENTING et al., 2009, MONTEDURO, 2013).
Os novos papéis da agricultura foram gradativamente sendo integrados em estratégias cada vez mais complexas voltadas a garantir sua interconectividade em prol do desenvolvimento rural (LEAKEY, 2010) e, com a crescente preocupação sobre as mudanças climáticas e o reconhecimento de nossa condição no antropoceno $^{1}$, cresce a necessidade de se adotar estratégias de integração entre as ciências ambientais e sociais e de incorporação do conhecimento local para fazer face à complexidade dos desafios a enfrentar (ALVES 2008; GARNELO; SANTOS, 2009; VAN DER LEEUW et al., 2011; NOBRE, 2012; ARTAXO, 2012) especialmente em situações que demandam planejamento para um futuro incerto, considerando a rapidez das taxas de mudanças ambientais induzidas pelo

\footnotetext{
1 Termo cunhado pelo Nobel Paul Crutzen para designar a era geológica atual resultante das interferências causadas pelo homem (CRUTZEN;STOERMER, 2000)
} 
homem e que exigem forte protagonismo das instituições, desde o nível local de comunidades, até o nível global, para garantir agilidade e condições de adaptação à nova realidade.

O termo sustenagilidade (do inglês sustainagility) vem sendo adotado para enfatizar a importância de desenvolver estratégias de capacidade adaptativa e transformativa, levando em consideração decisões (trade offs) em múltiplas escalas (JACKSON et al., 2010).

Esta realidade instigante que demanda reações imediatas da ciência traz, inevitavelmente, novos desafios ao já complexo quadro das ciências agrárias e daquelas a ela complementares, em particular ao se voltarem à reconhecidamente complexa realidade amazônica aonde, ao longo do último século, as atividades de pesquisa vêm evoluindo, de uma natureza praticamente monodisciplinar, para experiências multidisciplinares e interdisciplinares (HOMMA, 2003; COSTA, 2012a), com tendências a incorporar, de modo crescente, o caráter transdisciplinar para alcançar objetivos que demandem a integração de equipes de diversas disciplinas científicas com representantes de distintos segmentos externos ao meio científico (BERNARD; BUNING, 2013).
A pluralidade dos tipos de agriculturas encontrados na Amazônia e, consequentemente, dos atores sociais e culturais que as representam (COSTA, 2012b; ROBERT; LOPEZ, 2012) se constitui em um grande campo de aprendizado e inspiração para o delineamento e exercício da pesquisa agrícola e florestal interdisciplinar e transdisciplinar nesta região.

De imediato, há um amplo conjunto de políticas públicas vigentes ou em vias de serem implantadas, voltadas à sustentabilidade da atividade agrícola que necessitam, para a sua plena execução, de atividades de pesquisa e ação transdisciplinar, como é o caso daquelas voltadas a atividades agroecológicas constantes da Política Nacional de Agroecologia e de Produção Orgânica (PNAPO), considerando o caráter eminentemente transdisciplinar da prática agroecológica (MENDEZ et al., 2013; MONTEDURO, 2013), e o conjunto de iniciativas governamentais voltadas ao fortalecimento do extrativismo e das cadeias da sociobiodiversidade associadas ao manejo de florestas de uso múltiplo (CRUZ et al., 2011).

Este artigo aborda aspectos relacionados à tendência atual de exigir maior esforço interdisciplinar e 
transdisciplinar da pesquisa agropecuária na Amazônia para abordar um conjunto amplo e diversificado de grandes temas e políticas públicas voltadas ao atingimento da sustentabilidade regional.

\section{AGRICULTURAS AMAZÔNICAS}

As agriculturas amazônicas, em seu passado remoto, vêm sendo desvendadas por equipes de arqueólogos, que trazem à tona vários aspectos reveladores das formas de manejo dos recursos naturais, uso e conservação da biodiversidade, técnicas de melhoria na fertilidade do solo, bem como levantam pistas sobre a contribuição daquelas experiências à agricultura atualmente praticada em grande parte da região, integrada com a atividade extrativa e, mesmo, sobre estratégias a adotar atualmente (HOMMA, 2003; NEVES, 2006; SHEPARD; RAMIREZ, 2010).

A pluralidade das agriculturas vigentes vem sendo abordada por diferentes segmentos de autores, em geral agrupandoas nas categorias de agricultura patronal, agricultura familiar e da praticada por povos indígenas e comunidades tradicionais (HOMMA, 2003; MARTHA JUNIOR et al., 2011a).
Em particular, Costa (2009a, 2012b,c) vem oferecendo expressiva contribuição à compreensão da dinâmica recente do setor rural amazônico e, o conjunto de seis trajetórias tecnológicas apontadas por ele como aí prevalecentes, conseguem expressar, sob vários aspectos e características, grande parte da diversidade das agriculturas amazônicas. São três trajetórias voltadas aos sistemas camponeses (T1 que converge para pecuária de leite e cultivos permanentes; T2 que converge para sistemas agroflorestais e T3 que converge para pecuária de corte) e três trajetórias voltadas aos sistemas patronais (T4 que convergem para pecuária de corte; T5 de culturas permanentes e T6 de silvicultura), que foram analisadas quanto a um conjunto amplo de características socioeconômicas e ambientais (COSTA 2009a).

Em relação às agriculturas praticadas atualmente por povos indígenas $\mathrm{e}$ populações tradicionais, estão associadas à magnitude da sociodiversidade que a região abriga e, em grande medida, resultam do processo de povoamento de seu território (LIMA; POZZOBON, 2005; CAÑETE; BEGOT, 2010).

Nessa linha, destacam-se sistemas agrícolas, particularmente os agroflorestais, praticados por populações 
indígenas, atividades agrícolas e extrativistas praticadas por populações quilombolas e sistemas de produção alimentares adotados por populações ribeirinhas e populações residentes em reservas extrativistas (ADAMS et al., 2005; LIMA; POZZOBON, 2005; ALMEIDA, 2006; MILLER; NAIR, 2006; SIVIERO et al., 2009; FARIAS JUNIOR, 2011; NODA et al., 2012).

\section{A PESQUISA AGRÍCOLA NA AMAZÔNIA}

A trajetória da pesquisa científica oferecendo subsídios, inicialmente indiretos, à agricultura, remonta à era dos cientistas viajantes e de suas expedições, mas, a partir da criação do atual Museu Paraense Emílio Goeldi, no final do século 19 (FAULHABER; TOLEDO, 2001), esse passou a abrigar ações de pesquisa que abordavam aspectos relevantes para a agricultura e, só em 1939 foi criada uma instituição governamental voltada exclusivamente para a pesquisa agrícola, o Instituto Agronômico do Norte (IAN), vinculado ao Ministério da Agricultura, que foi o precursor da unidade da Empresa Brasileira de Pesquisa Agropecuária (Embrapa), ora denominada de Embrapa Amazônia Oriental, que iniciou suas atividades com ações voltadas, em particular, a espécies perenes amazônicas, especialmente seringueira e fruteiras, e ao manejo de várzeas para fins agrícolas (ALBUQUERQUE; LIBONATI, 1964; HOMMA, 2003: MARTHA JUNIOR et al., 2012b).

Posteriormente, foi criado outro instituto de pesquisa em Manaus, voltado à Amazônia Ocidental (IPEAOC). No que concerne ao uso racional e conservação florestal, embora tenha iniciado com os naturalistas ainda no inicio do século 20 com estudos sobre estrutura e composição, somente a partir da década de 1950, iniciaram os trabalhos de forma sistemática com interrupções e retomada nos anos 1970. Na Embrapa, a pesquisa florestal teve o seu marco histórico a partir de 1978 com a criação do Programa Nacional de Pesquisa Florestal (PNPF) (SUDAM, 1978 ; ENCONTRO ... 1991 ; YARED, 1996).

Com a criação da Embrapa, em abril de 1973, os dois institutos foram incorporados à nova estrutura e, ao longo dos anos seguintes, passaram por inúmeras transformações e, simultaneamente, outras unidades foram sendo criadas, chegando ao final da década anterior com um conjunto de seis centros agroflorestais sediados nos estados do Acre, Amapá, Amazonas, Pará, Rondônia e Roraima. Em termos gerais 
esses centros de pesquisa focam suas atividades em: (i) sustentabilidade dos recursos naturais, com redução do desmatamento, aproveitamento de áreas antropizadas e áreas degradadas; (ii) exploração sustentável da madeira de florestas naturais e reflorestamento; (iii) implementação de sistemas integrados de produção; (iv) agregação de valor aos produtos extrativos; (v) aumento de produtividade na produção de alimentos de origem animal e vegetal; (vi) busca de alternativas aos insumos convencionais; e (vii) consolidação da agricultura familiar (MARTHA JÚNIOR et al., 2011b).

Mais recentemente, a partir de 2009, foram criados centros de pesquisa da Embrapa, com mandatos distintos, nos três outros estados componentes da Amazônia Legal. São eles: Embrapa Agrossilvipastoril no Mato Grosso, Embrapa Pesca e Aquicultura em Tocantins e Embrapa Cocais no Maranhão.

Acompanhando a mudança gradual do paradigma da agricultura e de políticas públicas para o desenvolvimento da região, as equipes das unidades da Embrapa, incluindo as situadas na Amazônia, têm gradualmente evoluído de um perfil predominantemente de ciências agrárias para quadros mais diversificados, contemplando pessoal especializado nas áreas ambiental e de ciências sociais, o que vem ensejando uma também gradativa mudança na natureza dos projetos, que começam a contar com número crescente de atividades que envolvem estratégias de pesquisa transdisciplinar, para tratar temas cuja complexidade e natureza exigem este tipo de disciplinaridade.

Atuam complementarmente em pesquisa agrícola na região, institutos de pesquisa do Ministério da Ciência, Tecnologia e Inovação (MCTI), um conjunto de universidades que desenvolvem pesquisas direta ou indiretamente voltadas à agricultura e florestas, e algumas instituições não governamentais especializadas (COSTA, 2012a; MARTHA JUNIOR et al., 2012b), que ampliam assim as possibilidades de, em ações integradas, ampliar a prática da pesquisa transdisciplinar voltada às qustões regionais.

\section{PESQUISA INTERDISCIPLINAR E PESQUISA TRANSDISCIPLINAR: PECULIARIDADES E OPORTUNIDADES DE ADOÇÃO}

Para melhor compreensão do significado dos termos e percepção da sua importância à pesquisa agrícola da 
Amazônia, são apresentados breves esclarecimentos neste sentido.

A pesquisa interdisciplinar refere-se à integração de corpos discretos de conhecimento, com cada outro, para criar a síntese de um novo conhecimento (GRIFFIN et al., 2006). É uma forma de pesquisa realizada por equipes de indivíduos que integram informação, dados, técnicas, ferramentas, perspectivas, conceitos e/ou teorias de duas ou mais disciplinas ou corpos de conhecimento especializado para avançar no conhecimento fundamental ou para resolver problemas cujas soluções estão além do escopo de uma simples disciplina ou área de pesquisa (NATIONAL ACADEMY OF SCIENCE, 2005).

A pesquisa transdisciplinar é uma forma apropriada de pesquisa quando se busca soluções para problemas do mundo real, com alto grau de complexidade em termos de incertezas factuais, valores e atores sociais. Através de pontes entre diferentes componentes de conhecimento científico e social pode, significativamente, melhorar a qualidade, aceitação e sustentabilidade de tais soluções (WIESMANN et al., 2008)

A apresentação esquemática proposta por Bernard e Buning (2013), na Figura 1, permite compreender as diferenças no modo de integração do conhecimento entre equipes de diferentes abordagens disciplinares de pesquisa, incluindo as de caráter interdisciplinar e transdisciplinar.

A interdisciplinaridade refere-se à transferência de métodos de uma disciplina a outra (NICOLESCU, 2008), enquanto que a transdisciplinaridade, que diz respeito ao que está entre as disciplinas, através de diferentes disciplinas, além de todas as disciplinas, visando a compreensão do mundo presente, onde a unidade do conhecimento é imperativa (NICOLESCU, 2002), é um caráter essencial ao exercício pleno, por exemplo, da agroecologia, pois lhe confere a possibilidade de buscar soluções para problemas reais, com alto grau de complexidade em termos de incertezas factuais, valores e atores sociais, lançando mão de pontes entre diferentes componentes de conhecimento científico e social, permitindo melhorar a qualidade, aceitação e sustentabilidade das soluções propostas (WEISMANN et al., 2007), através de do engajamento ativo e de práticas aproximativas voltadas à solução construtiva de problemas (GIBBONS et al., 1994). 
Figura 1 - Representação esquemática das diferenças na integração do conhecimento (linhas pontilhadas) entre equipes de pesquisa mono, multi, inter e transdisciplinares.

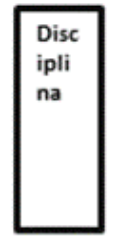

Monodisciplinar

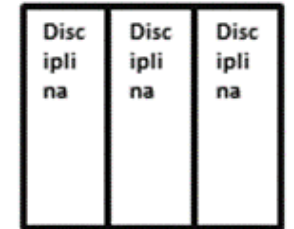

Multi-

disciplinar

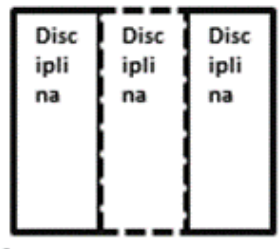

Inter-

disciplinar

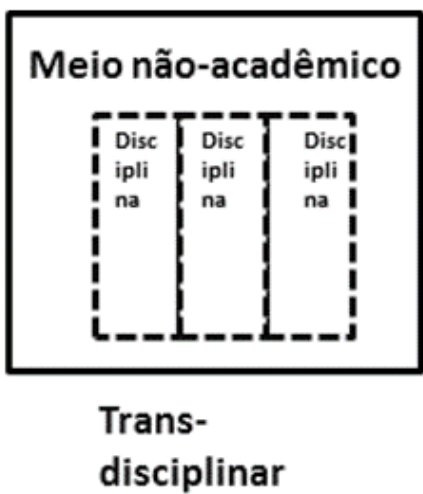

Fonte: BERNARD e BUNING (2013).

A transdisciplinaridade nasce da premente exigência de se consagrar o diálogo entre diferentes campos de saber sem impor o domínio de uns sobre os outros, acercando-se de uma atitude e postura que orientem a interação e a confiança entre os profissionais e seus conhecimentos (RODRIGUES, 2000).

É oportuno acrescentar que vem sendo reconhecido que vários dos problemas mundiais tidos como difíceis ou impossíveis de tratar, como é o caso do aquecimento global/mudança do clima e manutenção sustentável de suprimentos alimentícios, não podem ser compreendidos, manejados e resolvidos usando uma única ciência e , em particular, o desenvolvimento rural sustentável é identificado como se defrontando com grandes desafios de caráter econômico, social, ambiental e tecnológico que perpasssam limites disciplinares (HUNT et al., 2010), incluindo as diversidades de natureza das instituições envolvidas ,levando à necessidade de ampliar a adoção de estratégias interdisciplinates e transdisciplinares de pesquisa.

\section{A TRANSDISCIPLINARIDADE E} ALGUMAS QUESTÕES COMPLEXAS DO PANORAMA AGRÍCOLA AMAZÔNICO

No momento atual há um conjunto, significativo, de grandes questões globais ou nacionais com rebatimento regional, bem como questões associadas à aplicação de políticas públicas ou instrumentos legais 
que suscitam a atenção da pesquisa na tentativa de aplicar adequadamente as estratégias interdisciplinares e transdisciplinares.

A título de exemplo, podem ser citados os seguintes temas:

(i) As questões associadas às mudanças climáticas no que tange, em especial a estratégias de mitigação e adaptação requerem, ações de distintas disciplinas envolvendo grupos de interesse diversificados;

A adequação ambiental de estabelecimentos rurais, regulada pelo código florestal aprovado em 2012, demanda da pesquisa agrícola uma ampla gama de ações que, dado o caráter estreitamente relacionado a aspectos biofísicos e socioeconômicos das áreas a abordar, merece ser abordada via estratégias interdisciplinares e/ou transdisciplinares, conforme enfatiza Ferreira et al. (2012) ao se referir à necessidade de garantir uma maior integração entre tomadores de decisão e pesquisadores em ecologia, em temas associados à aplicação do código florestal;

(iii) As inúmeras demandas de pesquisa já expressas no Plano Nacional de Agroecologia e Produção Orgânica (PLANAPO), que demandam a aplicação da abordagem transdisciplinar, pelo próprio caráter da agroecologia, sua multidimensionalidade e a necessidade de interação com segmentos produtivos, em particular da agricultura familiar, povos indígenas e populações tradicionais, em ações participativas e transformadoras (MÉNDEZ et al., 2013);

(iv) As questões associadas à preocupação crescente com o impacto da implantação do Polo de Produção de Palma de Óleo (dendê) no estado do Pará, sobre o ambiente e sobre os contingentes de agricultores familiares da região, que pela sua complexa natureza e necessidade de lançar mão de disciplinas associadas a aspectos biofísicos, socioeconômicos, culturais e políticos, constitui-se, também, em tema complexo passível de abrigar iniciativas de pesquisa interdisciplinar e transdisciplinar;

(v) A agenda voltada a dar suporte à aplicação do Plano Nacional de Segurança Alimentar e Nutricional e a outros instrumentos relacionados à segurança e soberania alimentar constituem-se em atividades a demandarem ações de pesquisa, em especial transdisciplinar; Avanços de forma harmônica e integrada em ações do Programa Federal de Manejo Comunitário e Familiar (PMCF), instituido pelo Dec. 6.874/2009 no âmbito dos ministérios do 
Meio Ambiente e do Desenvolvimento Agrário;

$$
\text { O conjunto de demandas }
$$
associadas à atividade extrativista vegetal na Amazônia, contempladas no Plano Nacional de Promoção das Cadeias de Produtos da Sociobiodiversidade (PNPSB) e no Plano Nacional de Fortalecimento do Extrativismo (PLANAFE), em fase final de elaboração; e

(viii) As demandas voltadas à oferta de estratégias alternativas de cultivo sem o uso do fogo é também tema que merece intensificar a adoção de pesquisa transdisciplinar, dada a sua natureza de envolvimento dos segmentos produtivos, de modo participativo, ao longo da pesquisa.

Um fato recente que poderá ser um elemento positivo diferencial para as unidades da Embrapa situadas na Amazônia avançarem na práticas de pesquisa transdisciplinar, em vários temas de interesse da agricultura familiar, é o convênio firmado em nível nacional entre a Embrapa e a Confederação Nacional dos Trabalhadores na Agricultura (CONTAG), que já está iniciando a sua implementação na região Norte.

Finalizando, vale a pena atentar para uma demonstração patente da relevância de incorporar a abordagem transdisciplinar no trato de complexas questões regionais, expressa em Costa (2009b), ao discutir novos fundamentos para $\mathrm{o}$ desenvolvimento do setor rural da região Norte, onde enfatiza que uma nova institucionalidade se faz necessária, que permita um planejamento baseado no conhecimento e na participação, e que deverá fundamentar-se no melhor conhecimento possível sobre a sociedade e a economia amazônicas e sua base natural e, para tal, necessitará de capacidade própria, que lhes faculte o diálogo crítico atualizado com as concepções modernas de desenvolvimento que hoje pautam a pesquisa de fronteira das ciências da sociedade e das ciências naturais.

Enfatiza-se que, nesse esforço encontrar-se-ão as ferramentas para operar, valorizando as economias locais, discernindo quanto à diversidade dos seus sujeitos e ao "valor" e "usos" das disponibilidades e carências em termos de capitais físico, social, humano e natural, onde o conhecimento (sistêmico e dinâmico: complexo) que se cogita permitirá, ademais, o acompanhamento eficiente do desenvolvimento regional por indicadores que, por uma parte, contemplem as esferas econômica, social e ambiental e, por outra, permitam observar os níveis micro, meso e macrorregional. 
ALGUNS DESAFIOS, A GUISA DE CONCLUSÃO

Considerando válido à pesquisa agropecuária o que Nicolescu (1997) comentou a respeito da universidade, é válido comentar que se as instituições de pesquisa agrícola da Amazônia brasileira quiserem ser agentes reconhecidamente válidos do desenvolvimento sustentável têm, antes de tudo, que reconhecer a emergência de um novo tipo de conhecimento, denominado transdisciplinar, cuja produção implica a necessidade de uma abertura multidimensional: (i) em direção à sociedade civil; (ii) em direção a outros lugares de produção de um novo conhecimento (instituições privadas e empresas industriais e laboratórios, organizações sem fins lucrativos, etc.); (iii) em direção ao espaço-tempo cibernétivo; (iv) em direção à meta da instituição (no caso, da instituição de pesquisa e as demais associadas em redes), e (v) em direção a valores que regem sua própria existência.

Certamente, para garantir que a prática das abordagens de pesquisa interdisciplinar e transdisciplinar se amplie e se consagre na pesquisa agrícola e florestal na Amazônia, devem ser encarados alguns desafios de distintas ordens, incluindo: (i) contribuir à formação de pessoal com perfil compatível com as atividades interdisciplinares e transdisciplinares;(ii) ampliar o leque de perfis de formação profissional dos pesquisadores e assistentes, em especial nas ciências sociais; (iii) adotar estratégias de ampliação de parcerias inter e transdisciplinares garantindo maior diversidade de formação nas equipes de projetos de pesquisa; (iv) garantir estratégias de interação entre grupos de interesse; (v) adotar e aprimorar metodologias de pesquisa interdisciplinar e transdisciplinar; (vi) realizar eventos que abordem experiências em pesquisas inter e transdisciplinares; e (vii) aproveitar oportunidades de demandas de políticas públicas para ampliar o exercício da interdisciplinaridade $\quad$ e $\quad d a$ transdisciplinaridade, seja nas instituições de ensino, pesquisa, extensão e fomento, seja de instituições públicas, privadas e não-govermentais em suas diferentes esferas de responsabilidade e atuação.

Portanto, nos deparamos diante de cenário de desenvolvimento, cuja solução depende de um pensamento complexo e reformador se temos expectativas e a meta visão para atingirmos a sustentabilidade na região amazônica, 


\section{REFERÊNCIAS}

ADAMS, C., MURRIETA, R. S. S., SANCHES, R. A. Agricultura e Alimentação em Populações Ribeirinhas das Várzeas do Amazonas: novas perspectivas. Ambiente e Sociedade, Campinas, v. 8, n. 1, p. 1-23, 2005.

ALBUQUERQUE, M. de, LIBONATI, V. F. Ipean - 25 anos de pesquisa na Amazônia: histórico, organização, pesquisas. Belém: IPEAN, 1964.

ALMEIDA, A. W. B. De. Terra de quilombos, terras indígenas, "babaçuais livres", "castanhais do povo", faxinais e fundo de pasto: terras tradicionalmente ocupadas. Manaus: Projeto Nova Cartografia Social da Amazônia ; PPGSCUFAM ; Fundação Ford, 2006.

ALVES, D. S. Taking things public: a contribution to address human dimensions of environmental change. Philosophical Transactions of the Royal Society B, v. 363, p. 1903-1909, Feb., 2008.

ARTAXO, P. Break down boudaries in climate research. Nature. v. 481. p. 239, 2012.

BERNARD, M., BUNING, T. de C. Moving from monodisciplinarity to transdisciplonary: insights in barriers and facilitators that scientists faced during a interdisciplinary pig breeding program. In: INTERNATIONAL CONFERENCE ON ORGANIZATIONAL, LEARNING, KNOWLEDGE AND CAPABILITIES, 2013, Washington, D.C. Proceedings ... Washington, D.C., 2013.

CAÑETE, T. M. R.; BEGOT, L. H. Das populações pré-colombianas às populações tradicionais da Amazônia: a necessidade de dar voz ao nativo. In: ENCONTRO NACIONAL DE ESTUDOS
POPULACIONAIS, 13., 2010, Caxambu. Anais ... Caxambu, 2010.

COSTA, F. da A. Desenvolvimento agrário sustentável na Amazônia: trajetórias tecnológicas, estrutura fundiária e institucionalidade. In: BECKER, B., COSTA, F. DA A., COSTA, W. M. Um projeto para a Amazônia no século 21: desafios e contribuições. Brasília: CGEE, $2009^{a}$, p. 215-299.

Novos fundamentos institucionais para o desenvolvimento do setor rural na região norte. In: BECKER, B., COSTA, F. DA A., COSTA, W. M. Um projeto para a Amazônia no século 21: desafios e contribuições. Brasília: CGEE, p. 301-363, 2009b.

Ciência, tecnologia e sociedade na Amazônia. Belém: NAEA, 2012a. (Coleção Economia Política da Amazônia. Série III- Formação Histórica, v. 3).

política da Amazônia: historicidade, territorialidade, diversidade, sustentabilidade. Belém: NAEA, 2012b. (Coleção Economia Política da Amazônia. Série II: Fundamentos teóricometodológicos, v. 1)

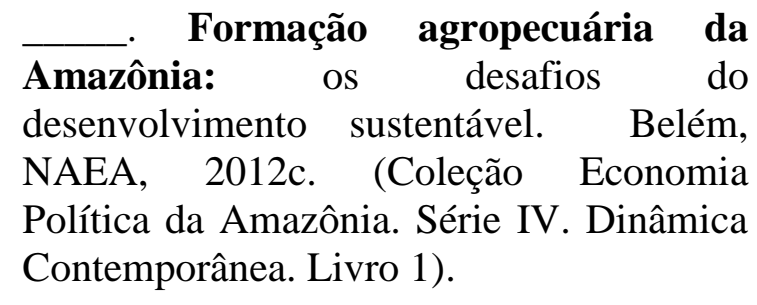

CRUTZEN, P. J., STOERMER, E. F. The "anthropocene". Global change Newsletter. V. 41, p. 12-13.

CRUZ, B.; SABLAYROLLES, P.; KANASHIRO, M.; AMARAL, M.; SIST, $P$ (org.). Relação empresa/comunidade no contexto do manejo florestal 
comunitário e familiar: uma contribuição do projeto "Floresta em Pé". Belém-PA: Ibama/DBFLO, 2011.

ENCONTRO SOBRE PESQUISA
TAPAJÓS, 1990, Santarém - PA. Documento final. Belém : EmbrapaCpatu: 1991, 69 p. (Embrapa-CPATU, Documentos, 55).

FARIAS JÚNIOR, E. de A. Práticas agrícolas e territorialidades dos quilombolas do Tambor. Agriculturas, v. 8, n. 4, p. 38-44, 2011.

FAULHABER, P.; TOLEDO, P. M. de (Eds.). Conhecimento e fronteira: história da ciência na Amazônia. Belém : MPEG, 2001. (Coleção Alexandre Rodrigues Ferreira).

FERREIRA, J.; PARDINI, R.; METZGE, J. P.; FONSECA, C. R.; POMPEU, P. S.; SPAROVEK, G.; LOUZADA, J. Towards environmentally sustainable agriculture in Brazil: challenges and opportunities for applied ecological research. Journal of Applied Ecology, v. 49, n. 3, p. 535-541, 2012.

GARNELO, L.; SANTOS, G. M. dos. A ciência contemporânea e o conhecimento indígena. GEEA: Caderno de Debates, Tomo 2, p. 67-114, 2009.

GIBBONS, M.; LIMOGES, C.; NOWOTNY, H.; SCHWARTZMAN, S.; SCOTT, P.; TROW, M. The new production of knowledge- the dynamics of science and research in contemporary societies. Sage, 1994.

GRIFFIN, G.; MEDHURST, P.; GREEN, $T$. Interdisciplinarity in interdisciplinary research programmes in the UK. University of Hull, May 2006.
HOMMA, A. K. O. História da agricultura na Amazônia: da era précolombiana ao terceiro milênio. Brasília: Embrapa, 2003.

HUNT, L.; BENGE, J.; CAMPBELL, H.; FAIRWEATHER, J.; GREER, G.; LUCOK, D.; MANHIRE, J.; MOLLER, H.; REID, J.; ROSIN, C.; SAUDERS, C. Experiences of transdisciplinarity in research on agricultural sustainability. Narratives of interdisciplinarity Studies of Farm Systems Sustainability. In: EUROPEAN IFSA SYMPOSIUM, $10^{\text {th }}$, 2010, Vienna. Proceedings ... Vienna, 2010. pp. 981-991.

JACKSON, L.; VAN NOORDWIJK, M.; BENGTSSON, J.; FOSTER, W.; LIPPER, L.; PULLEMAN, M.; SAID, M.; SNADDON, J.; VODOUHE, R. Biodiversity and agricultura sustainagility: from assessment to adaptative management. Current opinión in Environmental Sustainability, v. 2, p. 8087, 2010.

LEAKEY, R. R. B. Agroforestry: a delivery mechanism for multifunctional agriculture. In: KELLIMORE, L. R. Handbook on Agroforestry: Management Practices and Environmental Impact. New York : Nova Science Publishers, 2010, p. 461-471. (Environmental Science Engineering and Technology Series).

LIMA, D., POZZOBON, J. Amazônia socioambiental. Sustentabilidade e ecológica e diversidade social. Estudos Avançados, São Paulo, v. 19, n. 54, maio/ago., p. 45-76, 2005.

MARTHA JÚNIOR, G. B., CONTINI, E., NAVARRO, Z. Caracterização da Amazônia Legal e macrotendências do ambiente externo. Brasília: Embrapa Estudos e Capacitação, 2011a. 
(Documentos, Embrapa estudos e Capacitação, n. 1).

MARTHA JÚNIOR, G. B., CONTINI, E., PENA JÚNIOR, A. G. Arranjos institucionais para a Embrapa na Amazônia. Brasília: Embrapa Estudos e Capacitação, 2011b. (Documentos, Embrapa estudos e Capacitação, n.2).

MÉNDEZ, V. E.; BACON, C. M.; COHEN, R. Agroecology as a transdisciplinary, participatory, and actionoriented approach. Agroecology and Sustainable Food Systems, v. 37, p. 3-18, 2013.

MILLER, R. P.; NAIR, P. K. R. Indigenous agroforestry systems in Amazonia: from prehistory to today. Agroforestry Systems, v. 66, p. 151-164, 2006.

MONTEDURO, M. Environmental law and agroecology. Transdisciplinary approach to public ecosystem services as a new challenge for environmental legal doctrine, 22 European. Energy and Environmental Law Review, n. 1, p. 211, 2013.

NATIONAL ACADEMY OF SCIENCES. Facilitating transdisciplinar research. Washington: Committee on Facilitating Interdisciplinary Research Committee on Science, Engineering, and Public Policy, 2005.

NEVES, E. G. Arqueologia da Amazônia. Rio de Janeiro: Zahar, 2006.

NICOLESCU, B. A evolução transdisciplinar a universidade condição para o desenvolvimento sustentável. In: CONGRESSO INTERNACIONAL A RESPONSABILIDADE DA UNIVERSIDADE COM A SOCIEDADE,
1997, Bangkok. Anais ... Bangkok: IAUCU, 1997.

Manifesto of transdisciplinarity. New York: State University of New York Press, 2002.

Transdisciplinarity: theory and practice. [s.1.]: B. Nicolescu, 2008.

NOBRE, C. A. Unsustainable? Science, v. 336, p. 1361, jun. 2012.

NODA, S. N.; MARTINS, A. L. U.; SILVA, A. I. C.; BRAGA, M. D. S. Paisagens e etnoconhecimento na agricultura Ticuna e Cocama no alto rio Solimões, Amazonas. Boletim Museu Paraense Emilio Goeldi, v. 7, n. 2, p. 397416, 2012. (Se. Ciências Humanas).

RENTING, H.; ROSSING, W. A. H.; GROOT, J. C. J.; VAN DE PLOEG, J. D.; LAURENT, C.; PERRAUD, D. Exploring multifunctional agriculture. A review of conceptual approaches and prospects for an integrative transitional framework. Journal of Environmental Management, v. 90, p. S112-S123, 2009.

ROBERT, P., LOPEZ, C. Agriculturas amazônicas: cultivando plantas, saberes, paisagens e ideias. Boletim do Museu Paraense Emilio Goeldi, Ciências Humanas, v. 7, n. 2, p. 289-292, 2012

RODRIGUES, M. L. Caminhos da transdisciplinaridade: fugindo às injunções lineares. Serviço Social \& Sociedade, v. 64, p. 124-134, 2000.

SHEPARD JÚNIOR, G., RAMIREZ, H. "Made in Brazil": Human dispersal of the Brazilian nut (Bertholletia excelsa, Lecythidacea) in ancient Amazonia. Economic Botany, v. 65, n. 1, p. 44-65, 2010. 
SIVIERO, A., HAVERROTH, M., EVANGELISTA, R. Agricultura na reserva extrativista Cazumbá- Iracema, Acre. Revista Brasileira de Agroecologia, v. 4, n. 2, p. 1098- 1102, 2009.

SUDAM. Departamento de Recursos Naturais da. Estudo técnico de recomposição florestal em florestas de terra firme na Estação Experimental de Curuá-Una. Silvicultura, v. 14, n. 2, p. 442-443, 1978

VAN DER LEEUW, S.; COSTANZA, R.; AULENBACH, S.; BREWER, S.; BUREK, M.; CORNELL, S.; CRUMLEY, C.; DEARING, J. A.; DOWNY, C.; GRAUMLICH, L. J.; HECKBERT, S.; HEGMON, M.; HIBBARD, K.; JACKSON, S. T.; KUBISZEWSKI, I.; SINCLAIR, P.; SÖRLIN, S.; STEFFEN, W. Toward an Integrated History to Guide the Future. Ecology \& Society, v. 16, n. 4, Special section p1, 2011.

WIESMANN，U.; BIBER-KLEMM， S.; GROSSENBACHER-MANSUY, W.; HADORN, H. H.; HOFFMANN - RIEM, H.; JOYE, D.; POHL, C.; ZEMP, E. Enhancing Transdisciplinary Research: A Synthesis in Fifteen Propositions. In: HADORN, G. H.; HOFFMANN - RIEM, H.; $\quad$ BIBER - KLEMM, S.; GROSSENBACHER - MANSUY, W.; JOYE, D.; · POHL, C.; · WIESMANN, U.; ZEMP, E. (eds.) Handbook of Transdisciplinary Research. Amsterdam: Springer, 2008. pp. 433- 441, 2007.

YARED, J.A.G. Efeitos de sistemas silviculturais na florística e na estrutura de florestas secundárias e primária na Amazônia Oriental. 1996. 179 f. Tese (doutorado) - Universidade Federal de Viçosa, 1996. 